\title{
Management of disseminated intravascular coagulation: current insights on antithrombin and thrombomodulin treatments
}

REVIEW

This article was published in the following Dove Press journal:

Open Access Emergency Medicine

\section{Mineji Hayakawa}

Emergency and Critical Care Center, Hokkaido University Hospital, Sapporo, Japan
Correspondence: Mineji Hayakawa Emergency and Critical Care Center, Hokkaido University Hospital, NI4W5

Kita-ku, Sapporo 060-8648, Japan

Tel +8I I I 7067377

Fax +8 I II 7067378

Email mineji@dream.com

\begin{abstract}
Sepsis and septic shock are frequently complicated by disseminated intravascular coagulation (DIC), which decreases the survival rate of patients with sepsis. In the past, large international randomized controlled trials (RCTs) using physiological anticoagulants for sepsisinduced DIC were not performed; however, RCTs have been conducted for sepsis and/or septic shock. In these trials, physiological anticoagulants did not show any beneficial effects compared with placebo for the treatment of sepsis and/or septic shock. In Japan, DIC treatments using antithrombin (AT) and/or recombinant human soluble thrombomodulin (rhTM) are common for patients with sepsis-induced DIC. Recently, large propensity score analyses demonstrated that AT and rhTM improved survival in patients with sepsis-induced DIC. Furthermore, post hoc analyses and meta-analyses that selected patients with sepsis-induced DIC from the previous large international RCTs indicated that physiological anticoagulants improved survival without increasing the associated sepsis-induced DIC bleeding. DIC treatments, such as AT and rhTM, may demonstrate beneficial effects when they are targeted at patients with sepsisinduced DIC only.
\end{abstract}

Keywords: anticoagulant, critical illness, intensive care units, organ failure, sepsis

\section{Introduction}

Sepsis and septic shock are forms of systemic inflammatory and anti-inflammatory response syndrome, which can result in life-threatening organ dysfunction caused by severe infection. ${ }^{1}$ Sepsis and septic shock are frequently complicated by disseminated intravascular coagulation (DIC). ${ }^{2}$ Two decades ago, Rangel-Frausto et al reported that the frequency of DIC complication gradually increased as severity of sepsis increased. ${ }^{3}$ Recently, in Japan, several reports have indicated that half of the patients with sepsis and septic shock who were treated in intensive care units (ICUs) also had DIC. ${ }^{4-6}$ Furthermore, the mortality among patients with sepsis-induced DIC was higher than that among patients without sepsis-induced DIC (Figure 1). ${ }^{4-6}$

In the 2012 International Guidelines from the Surviving Sepsis Campaign, there are no details about the treatment of sepsis-induced DIC. ${ }^{7,8}$ However, in the guidelines newly published in $2016,{ }^{9,10}$ a chapter regarding anticoagulant therapies has been added, the target of which is general sepsis and not sepsis-induced DIC. ${ }^{9,10}$

In the present paper, we review the existing evidence regarding various treatments for sepsis-induced DIC and their efficacy. 


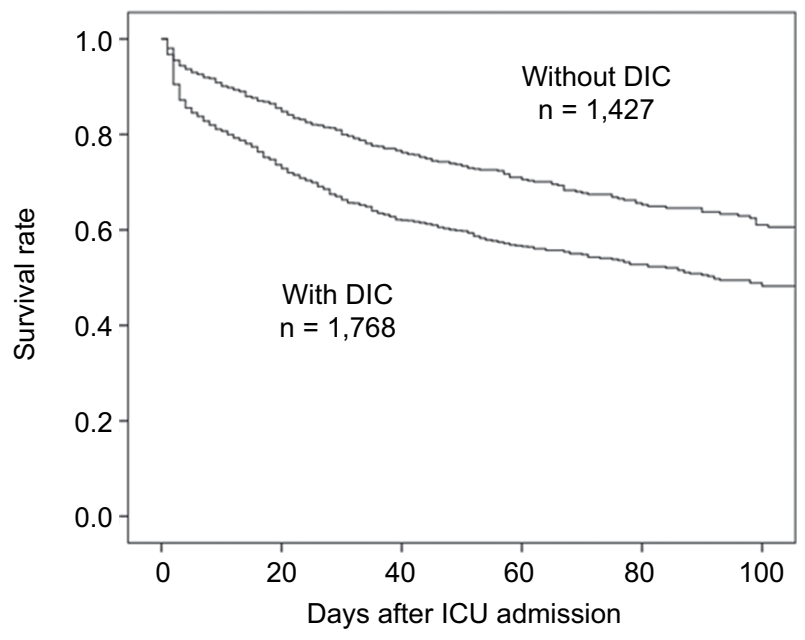

Figure I Survival rates for patients with and without sepsis-induced DIC. Notes: DIC was defined according to the Japanese Association for Acute Medicine DIC scores, ${ }^{49}$ as a score of $\geq 4$ on ICU admission. Reproduced from Hayakawa M, Saito S, Uchino S, et al. Characteristics, treatments, and outcomes of severe sepsis of 3I95 ICU-treated adult patients throughout Japan during 20I I-20I3. J Intensive Care. 2016;4:44. ${ }^{4}$

Abbreviations: DIC, disseminated intravascular coagulation; ICU, intensive care unit.

\section{Drugs available and used for the treatment of DIC in Japan and globally}

DIC treatment, which comprises various anticoagulants regardless of thrombosis prophylaxis, is one of the adjunct therapies for sepsis; however, the internationally recommended guideline for sepsis treatment does not suggest any specific treatment for DIC. ${ }^{9} 10$ In the Japan-Septic DIC study, which used a nationwide Japanese multicenter registry, 3,195 patients with sepsis were treated in the ICU from 2011 to 2013, half of whom had sepsis complicated by DIC. ${ }^{4}$ In almost all of these patients with sepsis complicated by DIC, anticoagulants were administered as DIC treatment. ${ }^{4}$ Based on the national administrative database in Japan, Murata et al also reported the use of drugs for sepsis-induced DIC from 2010 to $2012 .{ }^{11}$ In both of these studies, antithrombin (AT) and recombinant human soluble thrombomodulin (rhTM) were frequently used for the treatment of sepsis-induced DIC in Japan (Table 1). However, the frequencies of heparin treatment differed greatly between the two studies. ${ }^{4,11}$ Although the indications for heparin treatment were unclear for patients with sepsis-induced DIC in the study by Murata et al, ${ }^{11}$ they were specified in the Japan-Septic DIC study. ${ }^{4}$ In the report by Murata et al, heparin treatment was not administered solely for the treatment of sepsis-induced DIC. In other countries, treatment is rarely offered specifically for DIC. In a report which investigated an international sepsis registry including 12,881 patients with sepsis, treated in 276 ICUs in 37 countries from 2002 to 2005, AT treatment was provided for only $3 \%$ of the patients with sepsis (Table 1). ${ }^{12}$
Table I Drugs and frequency of DIC treatments

Japan-Septic DIC study, 3,195 patients with sepsis treated in ICU Any DIC treatments $\quad 47 \%$

Antithrombin $\quad 31 \%$

Thrombomodulin $27 \%$

Co-administration of antithrombin and $\quad 16 \%$

thrombomodulin

Protease inhibitors $\quad 12 \%$

Heparinoids $5 \%$

Japanese national administrative database, 14,324 patients with sepsis-induced DIC"

Antithrombin $\quad 42 \%$

Thrombomodulin $\quad 44 \%$

Protease inhibitors $\quad 11 \%$

Heparin $30 \%$

International sepsis registry, $12,88 \mathrm{I}$ patients with sepsis treated in $\mathrm{ICU}^{12}$

Antithrombin

$3 \%$

Abbreviations: DIC, disseminated intravascular coagulation; ICU, intensive care unit.

\section{Targeted anticoagulant treatments}

In the past two decades, several large randomized controlled trials (RCTs) using physiological anticoagulants (the KyberSept trial for AT, ${ }^{13}$ the OPTIMIST trial for recombinant tissue factor pathway inhibitor, ${ }^{14}$ the PROWESS [and PROWESSSHOCK, ADDRESS] study for recombinant human activated protein $C)^{15-17}$ were performed for sepsis and septic shock. None of these trials indicated the beneficial effects of treatment with physiological anticoagulants compared with the placebo. ${ }^{13-17}$ Furthermore, in a meta-analysis of RCTs, Freeman et al demonstrated that various physiological anticoagulant therapies did not improve treatment outcomes; rather, they were associated with significantly increased risk of bleeding complications among patients with sepsis and septic shock. ${ }^{18}$

In a post hoc analysis, where data of patients with sepsisinduced DIC were extracted from these large RCTs, anticoagulant therapies were found to improve patient outcomes. ${ }^{19,20}$ Recently, Umemura et al conducted a meta-analysis of RCTs which examined the efficacy and safety of anticoagulant therapy in patients with sepsis. ${ }^{21}$ The meta-analysis showed that although anticoagulant therapy resulted in no survival benefits and increased bleeding complications in the overall population of patients with sepsis, it improved survival without increasing the frequency of bleeding complications in patients with sepsis-induced DIC. ${ }^{21}$ Therefore, anticoagulant therapy was recommended for patients with sepsis-induced DIC only.

\section{Antithrombin}

AT is an important physiological anticoagulant, similar to protein $\mathrm{C}$ and thrombomodulin (TM), which is estimated to 
inhibit $80 \%$ of the coagulation activity against thrombin and various coagulation factors. ${ }^{22}$ However, in sepsis-induced DIC, AT activity is commonly decreased ${ }^{23-26}$ as a result of excessive thrombin generation, ${ }^{23}$ increased vascular permeability, ${ }^{24}$ degraded acceleration of AT, ${ }^{25}$ and impaired synthesis of AT in the liver. ${ }^{26}$ Decreased AT is associated with sepsis severity and high mortality. ${ }^{27,28}$

Recently, interaction between AT and vascular endothelial cells has been discussed. ${ }^{29}$ AT plays a role in the protection of endothelial cells by binding to the glycosaminoglycans, and suppressing capillary leakage. ${ }^{30}$ Furthermore, AT binding to the glycosaminoglycans exerts an anti-inflammatory effect in sepsis. ${ }^{31}$ Therefore, in septic patients with lower AT activity, AT supplementation is required to potentially improve outcomes.

The effects of high-dose AT treatment $(18,000 \mathrm{U} / 5$ days $-30,000 \mathrm{U} / 4$ days) in patients with sepsis were investigated in several RCTs. ${ }^{13,32-34}$ Some trials reported the beneficial effects of AT treatment among septic patients with decreased platelet counts ${ }^{32,34}$ and AT activity. ${ }^{33}$ The largest RCT (the KyberSept trial) demonstrated that high-dose AT therapy did not improve the mortality rate and increased the frequency of bleeding complications in patients with sepsis. ${ }^{13}$ However, a subgroup analysis of the KyberSept trial demonstrated that AT administration significantly decreased mortality rate in patients with sepsis-induced DIC and did not increase the frequency of bleeding complications. ${ }^{19}$ Furthermore, a recent meta-analysis of three RCTs by Umemura et al also indicated that AT treatment resulted in beneficial effects on mortality in patients with sepsisinduced DIC only. ${ }^{21}$

In Japan, septic DIC patients with AT activity $\leq 70 \%$ are clinically approved for AT supplementation therapy $(4,500$ U/3 days). Recently, several large propensity score analyses indicated that AT supplementation therapy decreased mortality rate in patients with sepsis-induced DIC. ${ }^{35-37}$ Based on the Japanese national administrative database, Tagami et al demonstrated that AT supplementation therapy decreased in-hospital mortality in patients with severe pneumonia ${ }^{35}$ and perforation of the lower intestinal tract. ${ }^{36}$ Also, in the JapanSeptic DIC study, AT supplementation therapy decreased early in-hospital mortality in patients with sepsis-induced DIC treated in the ICU. ${ }^{38}$ Furthermore, in patients with sepsis-induced DIC who had very low AT activity $(<45 \%)$, AT supplementation therapy provided survival benefits. ${ }^{39}$ However, optimal AT doses (high or low) as well as the target of keeping AT activity are still unclear.

\section{Recombinant human soluble thrombomodulin}

Physiological TM binds directly to thrombin with a high affinity, inhibiting thrombin activity and forming a thrombinTM complex. ${ }^{40}$ The TM-bound thrombin complex converts protein $\mathrm{C}$ into activated protein $\mathrm{C}$ which binds to protein $\mathrm{C}$ receptor on the vascular endothelial cell surface..$^{40}$ This prominent role of thrombin-TM complex in activating protein $\mathrm{C}^{41}$ is important in suppressing the coagulation and inflammatory systems. ${ }^{40}$

In Japan, rhTM was developed and approved for use clinically in 2008 after a Phase III RCT. ${ }^{42,43}$ Similar to the action of physiological TM on the vascular endothelial cell surface, rhTM has an active extracellular domain, can bind to thrombin, and can activate protein $\mathrm{C} .{ }^{43}$ Additionally, rhTM was shown to have a unique mechanism of action, in which thrombin generation is suppressed via activation of protein C without direct inhibition of thrombin activity, when used at therapeutic plasma concentrations. ${ }^{43}$

In a Phase III double-blind RCT performed in Japan, rhTM treatment improved DIC scores and reduced the frequency of bleeding symptoms in patients with DIC-associated sepsis and hematological malignancy more than heparin treatment. ${ }^{43}$ Furthermore, a post hoc analysis restricted to sepsis-induced DIC patients indicated a trend towards better outcomes in the rhTM group, compared to those in the heparin group. ${ }^{42}$ Some retrospective studies have indicated that rhTM reduced in-hospital mortality among patients with sepsis-induced DIC in clinical settings. ${ }^{38,44,45}$ In a Japanese nationwide multicenter registry study (Japan-Septic DIC study), propensity matching analysis demonstrated that rhTM significantly improved in-hospital all-cause survival without increasing the frequency of bleeding complications in the rhTM group (hazard ratio: 0.781 [95\% CI: 0.624-0.977, $P=0.030]$ ) (Figure 2). ${ }^{38}$

Although, in a Phase Ilb RCT conducted in Europe, rhTM treatment did not show direct survival benefits in overall septic patients with suspected DIC, the greatest survival benefit was observed in patients with respiratory or cardiac dysfunction and coagulopathy. ${ }^{46}$ Furthermore, in a metaanalysis which included three RCTs and nine observational cohort studies (not including the results of the Japan-Septic DIC study) ${ }^{38}$ rhTM treatment was associated with a reduced mortality trend among patients with sepsis-induced DIC. In conclusion, investigators recommended weekly rhTM treatment with moderate quality of evidence after evaluation using the Grading of Evidence, Assessment, Development and 


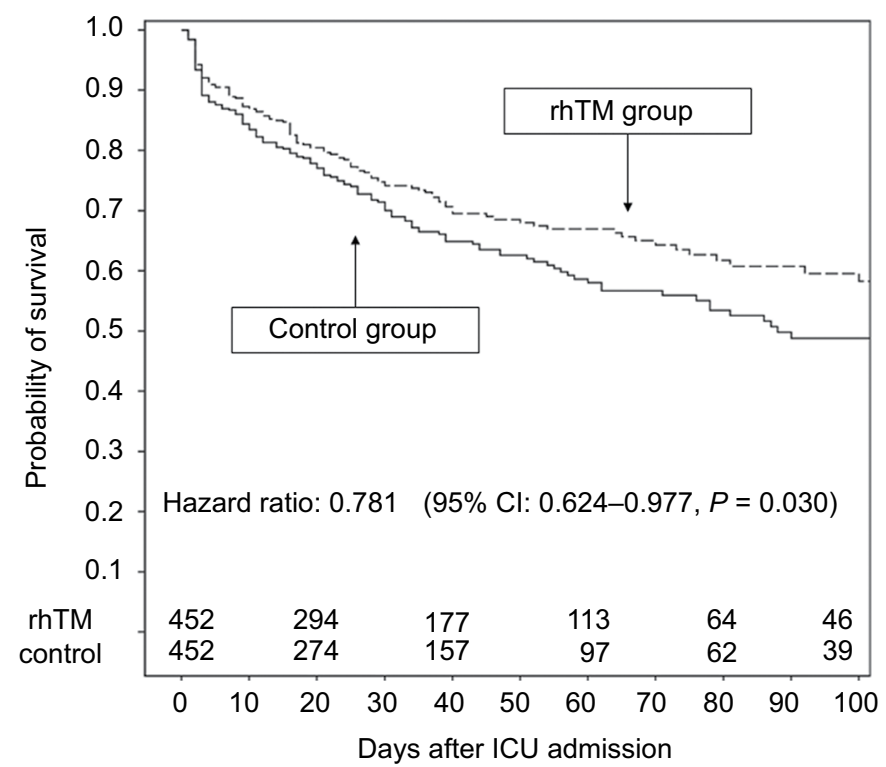

Figure 2 Survival rates for patients in the propensity score-matched control and rhTM groups.

Note: Reproduced from Hayakawa M, Yamakawa K, Saito S, et al. Recombinant human soluble thrombomodulin and mortality in sepsis-induced disseminated intravascular coagulation. A multicentre retrospective study. Thromb Haemost. 20 I6; I I5(6): I I57-I I66. ${ }^{38}$

Abbreviations: rhTM, recombinant human soluble thrombomodulin; ICU, intensive care unit.

Evaluation approach. ${ }^{47}$ Although rhTM is being widely used in clinical settings for treatment of DIC in Japan, ${ }^{4,11}$ rhTM is currently being evaluated by a Phase III international RCT in other countries. ${ }^{48}$

\section{Conclusion}

This paper reviewed the current treatment strategies for the management of sepsis-induced DIC. There are no existing guidelines with recommendations for the treatment of sepsis-induced DIC specifically. Although DIC treatments such as AT and rhTM are adjunct therapies for sepsis, they can demonstrate survival benefits when the treatment is provided to those with sepsis-induced DIC rather than all patients with sepsis.

\section{Disclosure}

M Hayakawa received a speaker's fee and research funding from Asahi Kasei Pharma Co. for an unrelated basic research project. The author reports no other conflicts of interest in this work.

\section{References}

1. Singer M, Deutschman CS, Seymour CW, et al. The Third International Consensus Definitions for Sepsis and Septic Shock (Sepsis-3). JAMA. 2016;315(8):801-810.

2. Levi M, Ten Cate H. Disseminated intravascular coagulation. $N$ Engl $J$ Med. 1999;341(8):586-592.

3. Rangel-Frausto MS, Pittet D, Costigan M, et al. The natural history of the systemic inflammatory response syndrome (SIRS). A prospective study. JAMA. 1995;273(2):117-123.
4. Hayakawa M, Saito S, Uchino S, et al. Characteristics, treatments, and outcomes of severe sepsis of 3195 ICU-treated adult patients throughout Japan during 2011-2013. J Intensive Care. 2016;4:44.

5. Ogura H, Gando S, Saitoh D, et al. Epidemiology of severe sepsis in Japanese intensive care units: a prospective multicenter study. $J$ Infect Chemother. 2014;20(3):157-162.

6. Matsuda N, Oda N, Aibiki M, et al. [2007 JSICM Sepsis 1st Registry: management of severe sepsis and septic shock in Japan]. J Jpn Soc Intensive Care Med. 2013;20:329-334. Japanese [with English abstract].

7. Dellinger RP, Levy MM, Rhodes A, et al. Surviving sepsis campaign: international guidelines for management of severe sepsis and septic shock: 2012. Crit Care Med. 2013;41(2):580-637.

8. Dellinger RP, Levy MM, Rhodes A, et al. Surviving Sepsis Campaign: international guidelines for management of severe sepsis and septic shock, 2012. Intensive Care Med. 2013;39(2):165-228.

9. Rhodes A, Evans LE, Alhazzani W, et al. Surviving Sepsis Campaign: international guidelines for management of sepsis and septic shock: 2016. Crit Care Med. 2017;45(3):486-552.

10. Rhodes A, Evans LE, Alhazzani W, et al. Surviving Sepsis Campaign: international guidelines for management of sepsis and septic shock: 2016. Intensive Care Med. 2017;43(3):304-377.

11. Murata A, Okamoto K, Mayumi T, Muramatsu K, Matsuda S. Recent change in treatment of disseminated intravascular coagulation in Japan: an epidemiological study based on a national administrative database. Clin Appl Thromb Hemost. 2016;22(1):21-27.

12. Beale R, Reinhart K, Brunkhorst FM, et al. Promoting Global Research Excellence in Severe Sepsis (PROGRESS): lessons from an international sepsis registry. Infection. 2009;37(3):222-232.

13. Warren BL, Eid A, Singer P, et al. Caring for the critically ill patient. High-dose antithrombin III in severe sepsis: a randomized controlled trial. JAMA. 2001;286(15):1869-1878.

14. Abraham E, Reinhart K, Opal S, et al. Efficacy and safety of tifacogin (recombinant tissue factor pathway inhibitor) in severe sepsis: a randomized controlled trial. JAMA. 2003;290(2):238-247.

15. Ranieri VM, Thompson BT, Barie PS, et al. Drotrecogin alfa (activated) in adults with septic shock. $N$ Engl J Med. 2012;366(22):2055-2064.

16. Abraham E, Laterre PF, Garg R, et al. Drotrecogin alfa (activated) for adults with severe sepsis and a low risk of death. NEngl J Med. 2005;353(13): 1332-1341. 
17. Bernard GR, Vincent JL, Laterre PF, et al. Efficacy and safety of recombinant human activated protein $\mathrm{C}$ for severe sepsis. $N$ Engl $J$ Med. 2001;344(10):699-709.

18. Freeman BD, Zehnbauer BA, Buchman TG. A meta-analysis of controlled trials of anticoagulant therapies in patients with sepsis. Shock. 2003;20(1):5-9.

19. Kienast J, Juers M, Wiedermann CJ, et al. Treatment effects of highdose antithrombin without concomitant heparin in patients with severe sepsis with or without disseminated intravascular coagulation. JThromb Haemost. 2006;4(1):90-97.

20. Dhainaut JF, Yan SB, Joyce DE, et al. Treatment effects of drotrecogin alfa (activated) in patients with severe sepsis with or without overt disseminated intravascular coagulation. J Thromb Haemost. 2004;2(11):1924-1933.

21. Umemura Y, Yamakawa K, Ogura H, et al. Efficacy and safety of anticoagulant therapy in three specific populations with sepsis: a meta-analysis of randomized controlled trials. JThromb Haemost. 2016;14(3):518-530.

22. Roemisch J, Gray E, Hoffmann JN, et al. Antithrombin: a new look at the actions of a serine protease inhibitor. Blood Coagul Fibrinolysis. 2002;13(8):657-670.

23. Opal SM, Kessler CM, Roemisch J, et al. Antithrombin, heparin, and heparan sulfate. Crit Care Med. 2002;30(5 Suppl):S325-S331.

24. Aibiki M, Fukuoka N, Umakoshi K, et al. Serum albumin levels anticipate antithrombin III activities before and after antithrombin III agent in critical patients with disseminated intravascular coagulation. Shock. 2007;27(2):139-144.

25. Seitz R, Wolf M, Egbring R, et al. The disturbance of hemostasis in septic shock: role of neutrophil elastase and thrombin, effects of antithrombin III and plasma substitution. Eur J Haematol. 1989;43(1):22-28.

26. Sie P, Letrenne E, Caranobe C, et al. Factor II related antigen and antithrombin III levels as indicators of liver failure in consumption coagulopathy. Thromb Haemost. 1982;47(3):218-220.

27. Fourrier F, Chopin C, Goudemand J, et al. Septic shock, multiple organ failure, and disseminated intravascular coagulation. Compared patterns of antithrombin III, protein $\mathrm{C}$, and protein $\mathrm{S}$ deficiencies. Chest. 1992;101(3):816-823.

28. Levi M, van der Poll $\mathrm{T}$. The role of natural anticoagulants in the pathogenesis and management of systemic activation of coagulation and inflammation in critically ill patients. Semin Thromb Hemost. 2008;34(5):459-468.

29. Chappell D, Brettner F, Doerfler N, et al. Protection of glycocalyx decreases platelet adhesion after ischaemia/reperfusion: an animal study. Eur J Anaesthesiol. 2014;31(9):474-481.

30. Iba T, Saitoh D. Efficacy of antithrombin in preclinical and clinical applications for sepsis-associated disseminated intravascular coagulation. J Intensive Care. 2014;2(1):66.

31. Iba T, Thachil J. Present and future of anticoagulant therapy using antithrombin and thrombomodulin for sepsis-associated disseminated intravascular coagulation: a perspective from Japan. Int J Hematol. 2016;103(3):253-261.

32. Inthorn D, Hoffmann JN, Hartl WH, et al. Antithrombin III supplementation in severe sepsis: beneficial effects on organ dysfunction. Shock. 1997;8(5):328-334.

33. Baudo F, Caimi TM, de Cataldo F, et al. Antithrombin III (ATIII) replacement therapy in patients with sepsis and/or postsurgical complications: a controlled double-blind, randomized, multicenter study. Intensive Care Med. 1998;24(4):336-342.
34. Eisele B, Lamy M, Thijs LG, et al. Antithrombin III in patients with severe sepsis. A randomized, placebo-controlled, double-blind multicenter trial plus a meta-analysis on all randomized, placebo-controlled, double-blind trials with antithrombin III in severe sepsis. Intensive Care Med. 1998;24(7):663-672.

35. Tagami T, Matsui H, Horiguchi H, et al. Antithrombin and mortality in severe pneumonia patients with sepsis-associated disseminated intravascular coagulation: an observational nationwide study. JThromb Haemost. 2014;12(9):1470-1479.

36. Tagami T, Matsui H, Fushimi K, et al. Supplemental dose of antithrombin use in disseminated intravascular coagulation patients after abdominal sepsis. Thromb Haemost. 2015;114(3):537-545.

37. Hayakawa M, Kudo D, Saito S, et al. Antithrombin supplementation and mortality in sepsis-induced disseminated intravascular coagulation: a multicenter retrospective observational study. Shock. 2016;46(6):623-631.

38. Hayakawa M, Yamakawa K, Saito S, et al. Recombinant human soluble thrombomodulin and mortality in sepsis-induced disseminated intravascular coagulation. A multicentre retrospective study. Thromb Haemost. 2016;115(6):1157-1166.

39. Iba T, Saitoh D, Wada H, et al. Efficacy and bleeding risk of antithrombin supplementation in septic disseminated intravascular coagulation: a secondary survey. Crit Care. 2014;18(5):497.

40. Weiler H. Regulation of inflammation by the protein C system. Crit Care Med. 2010;38(2 Suppl):S18-S25.

41. Esmon CT, Esmon NL, Harris KW. Complex formation between thrombin and thrombomodulin inhibits both thrombin-catalyzed fibrin formation and factor V activation. J Biol Chem. 1982;257(14):7944-7947.

42. Aikawa N, Shimazaki S, Yamamoto Y, et al. Thrombomodulin alfa in the treatment of infectious patients complicated by disseminated intravascular coagulation: subanalysis from the phase 3 trial. Shock. 2011;35(4):349-354.

43. Saito H, Maruyama I, Shimazaki S, et al. Efficacy and safety of recombinant human soluble thrombomodulin (ART-123) in disseminated intravascular coagulation: results of a phase III, randomized, doubleblind clinical trial. J Thromb Haemost. 2007;5(1):31-41.

44. Yamakawa K, Fujimi S, Mohri T, et al. Treatment effects of recombinant human soluble thrombomodulin in patients with severe sepsis: a historical control study. Crit Care. 2011;15(3):R123.

45. Yamakawa K, Ogura H, Fujimi S, et al. Recombinant human soluble thrombomodulin in sepsis-induced disseminated intravascular coagulation: a multicenter propensity score analysis. Intensive Care Med. 2013;39(4):644-652.

46. Vincent JL, Ramesh MK, Ernest D, et al. A randomized, double-blind, placebo-controlled, Phase $2 \mathrm{~b}$ study to evaluate the safety and efficacy of recombinant human soluble thrombomodulin, ART-123, in patients with sepsis and suspected disseminated intravascular coagulation. Crit Care Med. 2013;41(9):2069-2079.

47. Yamakawa $\mathrm{K}$, Aihara M, Ogura $\mathrm{H}$, et al. Recombinant human soluble thrombomodulin in severe sepsis: a systematic review and metaanalysis. J Thromb Haemost. 2015;13(4):508-519.

48. Phase 3 safety and efficacy study of ART-123 in subjects with severe sepsis and coagulopathy. Available from: https://clinicaltrials.gov/show/ NCT01598831. Accessed July 1, 2016.

49. Gando S, Iba T, Eguchi Y, et al. A multicenter, prospective validation of disseminated intravascular coagulation diagnostic criteria for critically ill patients: comparing current criteria. Crit Care Med. 2006;34(3):625-631.
Open Access Emergency Medicine

\section{Publish your work in this journal}

The Open Access Emergency Medicine is an international, peerreviewed, open access journal publishing original research, reports, editorials, reviews and commentaries on all aspects of emergency medicine. The manuscript management system is completely online and includes a very quick and fair peer-review system, which is all
Dovepress

easy to use. Visit http://www.dovepress.com/testimonials.php to read real quotes from published authors. 